\title{
Multilevel Analysis of Factors Associated with Left Behind Children in China
}

\author{
Yasuo Miyazaki ${ }^{1}$, Chenguang $\mathrm{Du}^{1}$, Joanna Papadopoulos ${ }^{1} \&$ Hongfei $\mathrm{Du}^{2}$ \\ ${ }^{1}$ School of Education, Virginia Tech, Blacksburg, US \\ 2 Department of Psychology, Guangzhou University, Guangzhou, China \\ Correspondence: Chenguang Du, 208 Turner Street, Blacksburg, VA, 24060, US. E-mail: dcheng6@vt.edu
}

Received: June 7, $2020 \quad$ Accepted: August 24, $2020 \quad$ Online Published: September 24, 2020

doi:10.5539/ass.v16n10p1 URL: https://doi.org/10.5539/ass.v16n10p1

\begin{abstract}
With the rapid development of urbanization in China, a growing number of rural workers migrate to urban cities for employment opportunities with leaving their children at home. These children are called left behind children (LBC) in China and their population has dramatically increased during the last 20 years. So far, many studies have examined what factors were associated with this increasing LBC populations. However, they were rarely guided by a holistic perspective. The current study investigated 1,691 left behind children in 166 communities using data from the China Health and Nutrition Survey (CHNS) in 2011. Based on the human ecology theory, this study explored family and contextual (community) characteristics associated with the left behind children (LBC) in China. The main results for this subpopulation of families with children revealed stark contrasts with the literature for the general population of migrants. That is, for the families with children, (1) contrary to the literature, father's education was negatively associated with the probability of LBC at the individual level, even after the income was controlled; (2) community average father's education was also negatively associated with LBC; but (3) community average household income was not associated with LBC once the average father's education was controlled. The policy implications of these results are briefly discussed.
\end{abstract}

Keywords: left behind children, rural to urban migration, family and community factors, multilevel logistic regression

\section{Introduction}

\subsection{Migrant Workers \& Left Behind Children in China}

The story, "Made in China" during the last quarter century was greatly contributed by the migrant workers toiling for substantial wages to produce exports (Chan, 2013). In fact, "rural migrant labor", was estimated to be $16.5 \%$ of total population in 2010, and has been the backbone of China's export industry since the mid-1990s (Ye \& Lu, 2011). For example, in coastal cities such as Shengzhen and Dongguan, the migrant population contributes to a majority of labor force $(70 \%$ to $80 \%)$ in the local industrial market. The rural-urban migration has also played a crucial role in China's epic urbanization within the last 20 years. In the 30 years since 1979 , China's urban population has grown by about 440 million to 622 million in 2009 (Chan, 2013). Among them, around 340 million could be attributable to the net migration (Ebenstein \& Zhao, 2015).

This rural to urban migration in China is different from internal migration in other countries. Although there are many temporary urban workers in China, not many of them can become permanent residents in cities because of the Chinese household registration system (hukou), (Kuang \& Liu, 2012). The "Hukou" system, which was modeled from the Soviet "propiska" (internal passport), translates literally as the "household registration system", but differs substantially from other systems of household registration (Chan \& Buckingham, 2008). It was initiated in 1958 and was intended to support the national industrialization strategy at the cost of the countryside development. At that time, this residency system was characterized by the rigid control of population flows into cities (Huang, 2014).

The current Chinese household registration system divides the household registration attribute into "agricultural hukou" and "non-agricultural hukou" (Ge, 2019). People with agricultural "hukou" are endowed with the rights to obtain land in rural areas, but are not allowed to enjoy social welfare in cities. This means these temporary migrant workers cannot obtain equal treatment for social welfare as received by their urban counterparts (Chan, 
2010). Neither do the family members of migrant labors to have any access to the social welfare system in cities. For example, the school-age children of migrant workers are not admitted freely to public schools in cities but are either rejected or required to pay an exorbitant extra fee (Jingzhong \& Lu, 2011). Therefore, many rural migrants do not have a better alternative, but to leave their children in the villages and hand the children's care over to their relatives. As a result, these children often endure long-term separation from their parents. This long-term separation from parents underwrites the widespread left-behind children phenomenon.

According to Guan and Deng (2019), 'Left Behind Children' (LBC) refers to the rural "hukou" children (under 16 years old) who have been left-behind at home by either both parents or one parent who has migrated while the other one does not have guardianship capacity. It was estimated that China already had 61 million left-behind children in rural areas in 2016, which was close to the total population of United Kingdom (Mimi Lau, 2016). More than one third of them even could not see their parents once a year, living totally without their parents' care and supervision (Wen \& Lin, 2012). Along with this growing population, many studies have showed that parental migration could potentially bring enduring adverse effects on LBC. They were very vulnerable to develop a series of psychological problems such as loneliness symptom, depression, anxiety and lower overall life satisfaction or happiness (Jia \& Tian, 2010; Fan, Su, Gill, \& Birmaher, 2010; Dai \& Chu, 2018). Compared to non-left behind children, the LBC often experience a higher rate of behavioral problems, such as more conduct problems, higher anti-social and aggressive behavior (Qu et al., 2018). In addition, LBC who are living in low-income situations have a higher risk of committing juvenile crimes (Shi-tan, 2009).

\subsection{Factors Associated with Left Behind Children}

Given the increasing concerns for the issues around LBC in China, many studies have been conducted to explore the reasons for the growing LBC population. So far, the dominant framework to interpret this phenomenon is based on the market mechanisms where such a migration is considered as product of a cost-benefit calculation (Borjas, 1993; Morawska, 2007). Under this perspective, many scholars reached an agreement that the income disparity between rural and urban areas will always be a primary incentive for rural surplus labors moving from agriculture work to the industry field at the cost of leaving their children behind (Fan, 2002; Liang \& Ma, 2004; Ye, Wang, Wu, He, \& Liu, 2013). Research even indicated that it is not necessarily the actual income that motivated laborers to migrate, but the optimistic expectations which may virtually outweigh any realistic increase in their income in terms of pushing them to move to cities (Gao \& Smyth, 2011).

Other studies indicated that beyond the economic benefits, the determinants on their migration could be divided into household and environmental factors. Among the household factors are gender, age, physical health, educational levels, and the household structure. For example, Roberts (2001) pointed out that unmarried labors were more likely than married couples to choose employment in distant regions or urban areas because the social culture expects the married couples to seek more stable life pattern. The marital effects on reducing the possibility of this migration, however, seem to be more salient among women than men in Chinese studies because some studies indicated that being married did not significantly reduce men's likelihood to participant in the labor migration (Yang \& Guo, 1999; Liang \& Chen, 2004). Furthermore, a study indicated that older rural workers tended to select off-farm employment near their home villages, while younger workers preferred remote regions (Yang, 2000). Household characteristics could also affect members' migration behavior. For example, the extended household structure such as presence of elderly parents, married children, or adult relatives were observed to increase the odds of participating in the migration because the parents and adult relatives can help with farm work and childcare while the migrants are away (Yang \& Guo, 1999). One of the most important factors from previous studies was migrants' educational level. Some studies indicated that rural laborers with relatively high educational levels were more likely to be employed outside of their home town, so they have a higher likelihood to migrate for urban employment. (Gao \& Smyth, 2011; Ye et al., 2013). Li and Zahniser (2002) even suggested that rural-to-urban migration is likely to increase in the future as the quality and quantity of education in rural areas improve.

As for the environmental factors, a body of studies showed that social network, local economy, and its population density will impact the decision of rural migration, which were often stated under the rubric of "push and pull" theory (Mejia, Pizurkim, \& Royston, 1979). For example, having relatives or friends in urban areas would work as a "pull" factor for the rural-urban migration because such social networks could help reduce the migration cost (Ye et al., 2013). In contrast, the community population density may work a "push" factor for migration because it is related to the degree of competition for local employment and social services (Zhang et al., 2015). Lower employment rates and income levels, less social security, and less farm land will also work together as a "push" factor (Willmore, Cao, \& Xin, 2012). 


\subsection{Theoretical Framework}

While the previous studies have provided some credible findings in explaining the formation of LBC, they were rarely guided by a proper theoretical framework. A major drawback is that they fail to explain the reasons of LBC from a holistic perspective. Most of the studies rely on a single-level analysis that focuses either only on the family / household levels (micro-level) or the community level (macro-level). However, a complete account of migration behavior should put the characteristics of family members under a broader community context (Massey, 1990). Any family member's decision to move or stay is a function of not only his / her motivations, but also the contextual environment where he /she reside. Therefore, the human ecological theory is an appropriate system theory to study the LBC phenomenon because it suggested that children do not develop in isolation, but within an interactive system between family and society (De Haas, 2010).

According to human ecology theory on migration and development, humans interact with their natural, socialcultural, and human-built environments to comprise a human ecosystem. For example, a family ecosystem consists of a family interacting with its environment which is defined both spatially and socially. The spatially and socially defined environment can be viewed as a hierarchy of systems at multiple levels moving from the most proximal to the most remote (Swain \& Garasky, 2007). In practice, both macrosystem and microsystem factors may contribute to the phenomenon of LBC. For example, the local economic condition mentioned before could be the institutional factor in the macrosystem to motivate family members to migrate. In contrast, the household income level in microsystem could also contribute to the migration of family members. To fully gain valuable information from multiple levels, we therefore adopt the multi-level analysis in our study.

At the child/family level, we paid close attention to the household income and father's educational attainment in the present study because, as aforementioned, the economic reasons were still a dominant incentive for the rural outmigration in most studies in China. We hypothesized that families with higher annual income were less likely to leave children behind. As for the father's educational attainment, we considered that father's educational attainment might play an important role on the family immigration decision because many rural families in China were still highly influenced by the patriarchal family culture. This traditional culture expects a husband to be a main financial sponsor and owns more marital power within the family especially when making important decisions for the whole family members. Some of the previous findings showed that male workers with higher education were more likely to migrate out even after marriage (Gao \& Smyth, 2011; Ye et al., 2013). This finding, however, was not consistent in other related studies (Chan \& Selden, 2014; Shao et al., 2016). Collecting all the above points together, we hypothesized that father's education attainment could be a significant factor in predicting the status of the left behind children with the direction of the impact left undetermined.

Other factors such as children's minority status, gender, and age, were also considered as the child/family level characteristics because they function together to compose the whole family ecosystem. Particularly, minority status was included to the list since some studies indicated that minority residents living in economically disadvantaged regions in China were more likely to migrate to work in urban areas (Fang, Yang, \& Meiyan, 2009; Li \& Zahnister, 2002).

At the community level, the predictors at the family level such as father's education, household income, and indicator of minority status were aggregated to form the predictors at the community level. Among them, the community average of father's education, and the community average household income were of our major interest since they were directly derived from the key variables of our interest at children/family level. The community mean minority status, which is the proportion of minority children that is approximately equivalent to the proportion of minority family in the community, was also of our interest. In addition, Qin (2010) found that the population of out-migrants were not evenly distributed because the inequality of the economic development of the different regions in China. Therefore, we included geographic region as a covariate for control to reflect the economic differences of the areas.

One of the major objectives of the present study is to fill some gap in the current LBC literature by examining the factors associated with this rural migration at the cost of leaving their children behind from the multilevel perspective. Results of the multilevel analysis could help address the following research questions: (1) what factors at family and community levels could play important roles for determining likelihood of becoming LBC. (2) how these factors at different levels work together to induce the LBC? Which factor has the strongest impacts? Answers from the multilevel perspective, which incorporate both family- and community-level factors would be very helpful in understanding the dynamic mechanism underlying this LBC phenomenon. 


\section{Method}

\subsection{Data and Measures}

The current study used data from the China Health and Nutrition Survey (CHNS). The CHNS is an ongoing international collaborative project between the University of North Carolina at Chapel Hill and the National Institute of Nutrition and Food Safety at the Chinese Center for Disease Control and Prevention (Chinese Center of Disease Control and Prevention, 2019). The information collected by this survey includes individual and household economic, demographic and social characteristics, and individual physical activity, nutrition intake and health status.

\subsection{Participant Characteristics}

The survey employed a multistage, random-clustered sampling procedure to draw a sample of about 4,400 households with about 19, 000 participants from over 200 communities or neighborhoods in nine provinces, with the first round conducted in 1989. To date, there were eleven wave of panel survey collected: 1989, 1991, 1993, 1997, 2000, 2004, 2006, 2009, and 2011.

We used the 2011 survey data in the current study because it is the most recent one and three municipal cities (Beijing, Shanghai, and Chongqing) were added to increase the geographical diversity, which were considered as the primary destination for these rural labor immigrants. Based on the definition of LBC (Guan \& Deng, 2019), we selected the children under the age of 18 and linked them to the household and community level datasets, the analytic sample had 1691 children in 166 communities. Note that communities represent villages and townships within the counties and urban/suburban neighborhoods within the cities and they were selected randomly after counties in nine provinces were stratified by the three levels of income level (Chinese Center of Disease Control and Prevention, 2019). Thus, counties were primary sampling units (PSUs) and households were secondary sampling units (SSUs).

\subsection{Descriptive Analysis}

The descriptive statistics for the child/family- and community-level characteristics used in the study were reported in Table 1.

Table 1. Descriptive statistics for data from year 2011 for the left behind children in China

Child/Family level $(n=1,691)$

\begin{tabular}{|c|c|c|c|c|c|}
\hline Categorical Variables (variable name) & \multicolumn{2}{|c|}{ Frequency } & \multicolumn{3}{|c|}{ Percent $(\%)$} \\
\hline \multicolumn{6}{|l|}{ Gender (dFemale $)$} \\
\hline Male (“0”) & \multicolumn{2}{|c|}{929} & \multicolumn{3}{|c|}{$54.9 \%$} \\
\hline Female (“1”) & \multicolumn{2}{|c|}{762} & \multicolumn{3}{|c|}{$45.1 \%$} \\
\hline \multicolumn{6}{|l|}{ Minority status (Minority) } \\
\hline Minority (“1”) & \multicolumn{2}{|c|}{254} & \multicolumn{3}{|c|}{$15.0 \%$} \\
\hline Majority (“0”) & \multicolumn{2}{|c|}{1437} & \multicolumn{3}{|c|}{$85.0 \%$} \\
\hline \multicolumn{6}{|l|}{ Status of Left Behind (Left_Behind) } \\
\hline Yes (“1”) & \multicolumn{2}{|c|}{603} & \multicolumn{3}{|c|}{$35.7 \%$} \\
\hline No (“0”) & \multicolumn{2}{|c|}{1088} & \multicolumn{3}{|c|}{$64.3 \%$} \\
\hline Continuous Variable (variable name) & $\mathrm{N}$ & Mean & $\mathrm{SD}$ & Minimum & Maximum \\
\hline Children's Age $(A g e)$ & 1691 & 7.73 & 4.79 & 0.00 & 18.00 \\
\hline Father's Educational Attainment $\left(F_{-} E d\right)$ & 1044 & 2.36 & 1.31 & 0.00 & 6.00 \\
\hline Household Gross Income (Log_Income) & 1665 & 10.27 & 1.32 & 0.00 & 13.34 \\
\hline \multicolumn{6}{|l|}{ Community Level $(n=166)$} \\
\hline Categorical Variables (variable name) & \multicolumn{2}{|c|}{ Frequency } & \multicolumn{3}{|c|}{ Percent $(\%)$} \\
\hline Municipality Area (Municipality) & \multicolumn{2}{|c|}{28} & \multicolumn{3}{|c|}{$16.9 \%$} \\
\hline Coastal Area (Coastal) & \multicolumn{2}{|c|}{28} & \multicolumn{3}{|c|}{$16.9 \%$} \\
\hline Inland Area (Inland) & \multicolumn{2}{|c|}{47} & \multicolumn{3}{|c|}{$28.3 \%$} \\
\hline Southwest Area (Southwest) & \multicolumn{2}{|c|}{32} & \multicolumn{3}{|c|}{$19.3 \%$} \\
\hline Northeast Area (Northeast) & \multicolumn{2}{|c|}{31} & \multicolumn{3}{|c|}{$18.7 \%$} \\
\hline Continuous Variable (variable name) & $\mathrm{N}$ & Mean & SD & Minimum & Maximum \\
\hline Minority Proportion (Mean_Minority) & 166 & 0.14 & 0.31 & 0.00 & 1.00 \\
\hline Mean Father's Educ. Attain. (Mean_F_Ed) & 156 & 2.27 & 1.01 & 0.00 & 5.00 \\
\hline Mean Gross Income (Mean_Inc) & 166 & 10.36 & 0.75 & 7.70 & 12.63 \\
\hline
\end{tabular}


The dependent variable ("Left Behind") was a binary variable coded either as "0" or "1" ("0" indicates non-left behind Children and "1" left behind Children). The proportion of LBC in the current dataset was $35.7 \%$ (see Table 1). At level 1 (child/family level), we used the children's gender (dFemale), Age (Age), minority status (Minority), father's educational attainment $\left(F_{-} E d\right)$, and Household Gross Income (Log_Income) as predicators. The variable "dFemale" is a dummy variable for child's gender, with " 0 " indicating male and " 1 " indicating "female". As shown in Table 1, $54.9 \%$ of children in the current sample were boys. The variable "Minority" is another dummy variable, indicating the minority status of a child such as "Hui", "Mongolian", "Tibetan", "Vaguer" and "Miao" etc. ("yes" coded as "1" and "no" coded as "0). As in Table 1, the reported minority children accounted for $15 \%$ of the entire sample. The variable "Age" is a continuous variable representing the children's age in 2011 , ranging from 0 to $18(M=7.73)$. The variable " $\log _{-}$Income" represents the natural logarithm of the total gross household income in "yuan" for each child. The use of log scale transformation was to alleviate the positive skewness in its distribution. The mean value of household income was 10.27, ranging from 0 to 13.34 in natural $\log$ scale. The last continuous variable is father's educational attainment $\left(F_{-} E d\right)$, which asked children's father about their "Highest Level of Education Attained". It was coded as six different levels with higher value indicating higher level of education: "0" represents "None"; " 1 " represents "primary school"; "2" represents "lower middle school", "3" represents "higher middle school", "4" represents "Technical or vocational degree"; "5" represents "University or college degree"; "6" represents "Master's degree or higher". The average value of father's education is 2.36 , meaning that the average father's degree in current study is in between middle and high school level.

Level 2 (community level) variables include: Municipality/Geographic regions (categorical), the proportion of minority within community (Mean_Minority, $M=0.14$ ), the average father's educational attainment (Mean_F_Ed, $M=2.27$ ) and the mean community income in natural $\log$ scale (Mean_Inc, $M=10.36$ ). The Municipality/Geographic regions consists of five categories: Municipal cities (Municipality $n=28$ (16.9\%)), Costal Area (Coastal, $n=28(16.9 \%)$ ), Inland Area (Inland, $n=47(28.3 \%)$ ), Southwest Area (Southwest, $n=32$ $(19.3 \%)$ ), and Northeast Area (Northeast, $n=31(18.7 \%)$. It should be noted that the geographic region was created by decomposing and recoding the category variable "province" in current dataset based on the previous study (Tong \& Piotrowsk, 2012). For example, the variable Coastal was created by combining the Jiangsu and Shangdong provinces, and the Inland was created by combining Henan, Hubei and Hunan provinces. We created four dummy variables for the five categories of Municipality/Geographic variable, and left the Southeast area as the reference group in our HLM analysis. The remaining three variables, Mean_F_Ed, Mean_Income, and Mean_Minority were created as the average of the corresponding individual level variables.

\subsection{HGLM Analysis}

Our major purpose of current study is to examine what are the reasons for parents to migrate by leaving their children behind. In order to answer the research questions, a multilevel logistic regression model (MLRM) was utilized since the dependent variable, Left_Behind, was a binary variable and the data had nested data structure (i.e., children (family) nested within communities). The MLRM is one form of hierarchical generalized linear model (HGLM) for discrete outcome variables and it is used for binary outcomes with logit link function. The link function is a nonlinear transformation of the expected value (or mean) of outcome variable $(Y)$ in a convenient form and the logit is the form of transformation for the binary outcomes. In addition, the transformed mean will be used as the dependent variable for the subsequent structural regression model (see Raudenbush \& Bryk, 2002 for more detail for HGLM). The analysis was conducted following the concept of taxonomy of statistical models (Singer \& Willet, 2003), which represents a set of relevant models that address the research questions of interest. All the HGLM analysis was conducted by HLM version 7 software (Raudenbush et al., 2011). The odds ratios are used as indicators of effect sizes, with the cut-off for large practical significance being an odds ratio greater than 2.0 (or less than 0.5, for negative relationships (Monahan, McHorney, Stump, \& Perkins, 2007). The odds-ratios will be used for interpreting the effect size in the later section.

First, an unconditional model (Model 1) which does not include any predictors at both levels, was used to fit the data as the baseline model to examine the amount of variability among communities. Since HGLM takes a form of a set of sampling model, link function, and structural model, the level 1 model in Model 1 was specified in the following equations:

Model 1.

Level 1:

Sampling model: $\quad$ Prob $\left(\right.$ Left_Behind $\left._{\mathrm{ij}}=1 \mid \beta_{j}\right)=\phi_{i j}$

Link function: $\quad \log \left[\phi_{i j} /\left(1-\phi_{i j}\right)\right]=\eta_{i j}$

Structural model $\quad \eta_{i j}=\beta_{0 j}$ 
where the sampling model equation indicates that the dichotomous outcome variable Left_Behind has a Bernoulli distribution with parameter $\phi_{i j}$, which is the probability of a child $i$ in community $j$ will become the left behind child; the link function equation indicates that the link function for the Bernoulli distribution is the logit link where $\eta_{i j}$ is the log-odds (i.e., logit), which is the natural logarithm of odds $\phi_{i j} /\left(1-\phi_{i j}\right)$; and the structural model equation states that the $\log$-odds for child $i$ in community $j$ is simply represented as the logit probability of the community $j$ to which the child $i$ belongs.

At level 2, the community $j$ 's average logit $\left(\beta_{0 j}\right)$ becomes the outcome variable as the sum of overall average $\left(\gamma_{00}\right)$ and the unique effect of community $j\left(u_{0 j}\right)$. That is,

Level 2:

$$
\beta_{0 j}=\gamma_{00}+u_{0 j}
$$

where $u_{0 j}$ is the level 2 random error that represents the unique effect of community $j$, and is assumed to be independent, identically distributed (i.i.d.) as normal distribution with mean of 0 and variance $\tau_{00}$, i.e., $u_{0 j} \sim$ i.i.d. $N\left(0, \tau_{00}\right)$.

After confirming that there is significant variability of the logits of LBC across community (i.e., rejecting $\mathrm{H}_{0}$ : $\tau_{00}=0$ ), which we will show in the results section, we proceeded to the next step. In this step (Model 2), children level (level 1) variables were added to the model for the purpose of better understand how the logit probability is associated with child and family characteristics. The level 1 predictors that were included were: child's gender (dFemale), Age, Minority, father's educational attainment $\left(F \_E d\right)$ and household gross income (Log_Income). Continuous variables such as $A g e, F \_E d$, and $L o g \_I n c o m e$ were group mean centered in order to capture the within community between child/family's variability (Allison, 2009). For this model, the intercept $\left(\beta_{0 j}\right)$ was specified as random and all the slopes were fixed after confirming them using the deviance test.

\section{Model 2.}

Level-1 Model:

$$
\begin{aligned}
& \text { Prob }\left(\text { Left_Behind }_{i j}=1 \mid \beta_{j}\right)=\phi_{i j} \\
& \begin{array}{l}
\log \left[\phi_{i j} /\left(1-\phi_{i j}\right)\right]=\eta_{i j} \\
\eta_{i j}=\beta_{0 j}+\beta_{1 j} *\left(\text { Age }_{i j}\right)+\beta_{2 j} *\left(\text { dFemale }_{i j}\right)+\beta_{3 j} *\left(\text { Minority }_{i j}\right) \\
\quad+\beta_{4 j} *\left(F \_E d_{i j}\right)+\beta_{5 j} *\left(\text { Log_Income }_{i j}\right)
\end{array}
\end{aligned}
$$

Level-2 Model:

$$
\begin{aligned}
& \beta_{0 j}=\gamma_{00}+u_{0 j} \\
& \beta_{1 j}=\gamma_{10} \\
& \beta_{2 j}=\gamma_{20} \\
& \beta_{3 j}=\gamma_{30} \\
& \beta_{4 j}=\gamma_{40} \\
& \beta_{5 j}=\gamma_{50} .
\end{aligned}
$$

Note that in Model 2, the intercept $\beta_{0 j}$ now represents the adjusted mean logit probability of LBC for community $j$ after adjusting for Age, dFemale, Minority, $F \_E d$, and Log_Income accordingly, the grand mean $\gamma_{00}$ now represents the overall adjusted grand mean logit probability of LBC.

In the third step, in order to better understand the significant variability of likelihood of LBC among communities found both in Model 1 and Model 2, we added the compositional variables such as Mean_Minority, Mean_F_Ed, and Mean_Income to the level 2 model for the level 1 intercept $\left(\beta_{0 j}\right)$. These compositional variables represent certain community characteristics, and they are frequently used to elucidate the contrast between the effects of these compositional variables and those of the corresponding individual level as the compositional or contextual effects in multilevel modeling literature.

Thus, as Model 3, a compositional/contextual effects model (Raudenbush \& Bryk, 2002, pp. 139 - 141) was specified as follows.

\section{Model 3.}

Level-1 Model:

$$
\begin{aligned}
& \operatorname{Prob}\left(\text { Left_Behind }_{i j}=1 \mid \beta_{j}\right)=\phi_{i j} \\
& \begin{array}{l}
\log \left[\phi_{i j} /\left(1-\phi_{i j}\right)\right]=\eta_{i j} . \\
\eta_{i j}=\beta_{0 j}+\beta_{1 j}^{*}\left(\text { Age }_{i j}\right)+\beta_{2 j} *\left(\text { dFemale }_{i j}\right) \\
\quad+\beta_{3 j} *\left(\text { Minority }_{i j}\right)+\beta_{4 j} *\left(F_{-} E d_{i j}\right)+\beta_{5 j} *\left(\text { Log_Income }_{i j}\right)
\end{array}
\end{aligned}
$$


Level-2 Model:

$$
\begin{aligned}
\beta_{0 j}= & \gamma_{00}+\gamma_{01} *\left(\text { Mean_Minority } y_{\mathrm{j}}\right)+\gamma_{02} *\left(\text { Mean_F_Ed } d_{\mathrm{j}}\right) \\
& \quad+\gamma_{03} *(\text { Mean_Income } \mathrm{j})+u_{0 \mathrm{j}} \\
\beta_{1 j}= & \gamma_{10} \\
\beta_{2 j}= & \gamma_{20} \\
\beta_{3 j}= & \gamma_{30} \\
\beta_{4 j}= & \gamma_{40} \\
\beta_{5 j}= & \gamma_{50} .
\end{aligned}
$$

Note that, for Model 3, the intercept $\left(\beta_{0 j}\right)$ was specified as random and all the slopes were kept fixed as was done in Model 2.

Model 4 builds on Model 3 by adding four geographic dummy variables (Municipality, Costal, Inland, and Northeast) at level 2 to see whether different regions could impact the intercept of level 1 model after controlling for other variables. Southwest region (Southwest) was used as reference region. Again, similar to Model 2 and

\begin{tabular}{|c|c|c|c|c|c|}
\hline & $\begin{array}{c}\text { Model } 1 \\
\text { (Unconditional } \\
\text { Model) }\end{array}$ & $\begin{array}{c}\text { Model } 2 \\
\text { (Level } 1 \\
\text { Predictor Added) }\end{array}$ & $\begin{array}{c}\text { Mode3 } \\
\text { (Compositional } \\
\text { Effect Model) }\end{array}$ & $\begin{array}{l}\text { Model } 4 \\
\text { (Geographic } \\
\text { Region Added) }\end{array}$ & $\begin{array}{c}\text { Standardized } \\
\text { Odds Ratio } \\
\text { for Model } 4\end{array}$ \\
\hline \multicolumn{6}{|l|}{ Fixed Effects } \\
\hline Intercept, $\gamma_{00}$ & $-0.858^{* * * *}(0.126)$ & $-4.423^{* * *}(0.471)$ & $-4.034^{* * *}(0.414)$ & $-2.958^{* * *}(0.456)$ & \\
\hline Age,$\gamma_{10}$ & & $0.054(0.034)$ & $0.050(0.033)$ & $0.043(0.032)$ & 1.229 \\
\hline dFemale, $\gamma_{20}$ & & $0.346(0.304)$ & $0.195(0.302)$ & $0.203(0.301)$ & 1.225 \\
\hline Minority, $\gamma_{30}$ & & $1.811(1.081)$ & $1.654(1.067)$ & $1.538(1.023)$ & 4.655 \\
\hline$F_{-} E d, \gamma_{40}$ & & $-0.387^{*}(0.184)$ & $-0.446^{*}(0.193)$ & $-0.452^{*}(0.195)$ & 0.553 \\
\hline Log_Income, $\gamma_{50}$ & & $-0.563^{* * *}(0.140)$ & $-0.513^{* * * *}(0.138)$ & $-0.482^{*}(0.135)$ & 0.529 \\
\hline Mean_Minority, $\gamma_{01}$ & & & $1.470^{*}(0.615)$ & $0.715(0.625)$ & 1.248 \\
\hline$M e a n_{-} F_{-} E d, \gamma_{02}$ & & & $-1.012^{* *}(0.308)$ & $-0.977^{* * *}(0.311)$ & 0.373 \\
\hline Mean_Income, $\gamma_{03}$ & & & $-0.002(0.375)$ & $0.2372(0.351)$ & 1.195 \\
\hline Municipality, $\gamma_{04}$ & & & & $-1.409(0.750)$ & 0.244 \\
\hline Coastal, $\gamma_{05}$ & & & & $-1.729^{*}(0.836)$ & 0.177 \\
\hline Inland, $\gamma_{06}$ & & & & $-0.936(0.583)$ & 0.392 \\
\hline Northeast, $\gamma_{07}$ & & & & $-2.003^{*}(0.809)$ & 0.135 \\
\hline \multicolumn{6}{|l|}{ Variance Components } \\
\hline Community mean & $1.654^{* * * *}(0.318)$ & $3.765^{* * * *}(1.323)$ & $2.124^{* *}(0.860)$ & $1.663 * *(0.768)$ & \\
\hline Pseudo $\mathrm{R}^{2}$ for $\mathrm{L} 2$ Variance & & Base & $44.6 \%$ & $57.2 \%$ & \\
\hline
\end{tabular}
Model 3, only the level 1 intercept was allowed to vary from community to community and the other level 1 slopes were fixed.

\section{Results}

The results of the unconditional model (Model 1) indicated that the estimated average log odds of left behind children was -0.858 , which was statistically significantly different from zero at 0.01 level (see Table 2 ).

Table 2. Results of fitting a taxonomy of HGLMs

${ }^{* * *} p<0.001,{ }^{* *} p<0.01,{ }^{*} p<0.05$; Standard error associated with its point estimate is in parentheses; Standardized odds ratio represents the odds ratios for one standard deviation change for continuous independent variables and the regular odds ratios (i.e., for one-unit change) for dichotomous variables.

This is equivalent to the probability of $1 /[1+\exp (-0.858)]=0.298$, which corresponds to the observed proportion, 0.356 (Note 1) (Table 1). Meanwhile, the estimated between community variance $\left(\tau_{00}\right)$ was $1.654($ s.e. $=0.318$ ), which was statistically significant at the 0.01 level (see Table 2). This indicates that there is a significant variability of the logit probability of LBC among communities, further justifying the use of HGLM in our study. The variance 1.654 (or standard deviation $(S D)=\sqrt{\tau_{00}}=1.286$ ) implies that $95 \%$ of the community mean logit 
of LBC approximately lied between $[-3.379,1.663]$, which is $[0.03,0.84]$ in probability, indicating that the LBC rates vary substantively from community to community.

The results of Model 2 showed that the except Age, dFemale, and Minority $\left(\gamma_{10}=0.054, p>0.05 ; \gamma_{20}=0.346, p>\right.$ 0.05 ; and $\gamma_{30}=1.811, p>0.5$ respectively), all the other predictors at level 1 were statistically significant. To be more specific, the results showed that the household gross income and father's educational attainment were statistically significant at 0.05 level, and both variables had negative effects on the likelihood of LBC, controlling for other variables $\left(\gamma_{40}=-0.387, p<0.05\right.$ for $F_{-} E d$ and $\gamma_{50}=-0.563, p<0.01$ for Log_Income). This means that the higher the fathers' educational attainment and gross income, the less likely the children would be left behind.

The results of Model 3 indicated that compositional variables did explain the variation of the probability of LBC among communities. That is, $44.6 \%$ of the between-communities variance were explained after three aggregated variables ("Mean_Minority", "Mean_F_Ed", and "Mean_Income") were added as predictors for Level 1 intercept $\beta_{0 j}$ (see Pseudo-R ${ }^{2}$ for L2 Variance in Table 2). Specifically, mean father's education attainment (Mean_F_Ed) was negatively related to the logit probability of left behind children $\left(\gamma_{02}=-1.012, p<0.01\right)$. Mean_Minority was also statistically significant in predicting the level 1 intercept $\left(\gamma_{01}=1.470, p<0.05\right)$, indicating that on average, the community with higher proportion of minority children tend to have higher probability of LBC. On the other hand, the mean family income (Mean_Income) was not a statistically significant predictor for the adjusted community logit mean probability.

The results for Model 4 (final model) showed that there was significant difference on the probability of becoming LBC among five municipality/geographic regions even after the community context variables were controlled for. Specifically, compared to the reference region, which is Southwest, all the other regions had lower probability of LBC. Especially, the salient difference was observed between the Northeast and Southwest regions $\left(\gamma_{04}=-2.003, p<0.05\right)$, as well as between Costal and Southwest regions $\left(\gamma_{02}=-1.729, p<0.05\right)$. It means that on average, children in Southwest had 0.135 or 0.177 times less likely to be left behind in odds than those in the Northeast or Costal regions respectively (for these odds-ratios, they can be obtained by simply exponentiating the coefficients. That is, $\exp (-2.003)=0.135$ and $\exp (-1.729)=0.177$ and these are reported under the column of Standardized Odds ratio in Table 2). These values are considered to be large effects according to the rule of thumb (Monahan, McHorney, Stump, \& Perkins, 2007). Although we did not find a significant difference between Municipality and Southwest, the general trend was that children in municipality regions are 0.244 times less likely to be left behind in terms of odds compared to Southeast regions, which was a large effect in practice again. In terms of the variance-accounted-for measure, after the geographic dummy variables were added, the pseudo- $\mathrm{R}^{2}$ increased to $57.2 \%$ in Model 4 from $44.6 \%$ in Model 3, indicating that additional $12.6 \%$ of the variance in Model 2 was explained by adding the municipality/geographical region information.

\subsection{Odds-ratio and Standardized Odds-ratio}

As mentioned in HGLM analysis section and already used in describing the region effects above, odds-ratios (ORs) are commonly used to describe the effect size (ES) and it is useful to discuss practical and substantive significance. OR for dichotomous independent variable can be immediately obtained by exponentiating the estimated regression coefficients as was done for dummy variables for region above. This applies to minority status in Model 4. Though it was not statistically significant at 0.05 level, the OR was 4.655 as in Table 2. This means that when other things equal minority children were 4.655 times more likely to be LBC in odds compared to majority children, which was extremely large effect and was the largest among all independent variables in Model 4.

When it comes to continuous independent variables, we need to standardize the regular OR if we want to compare the ESs among different variables. This can be done by only standardizing the scale of independent variables, that is, the standardized OR (SOR) can be obtained by multiplying the SD of the independent variable given in Table 1 to the estimated coefficient and then exponentiate it (Kaufman, 1996) (Note 2). The right most column of Table 2 reports the SOR for the continuous independent variables in Model 4. From Table 2, we can see that at the child/family level, the SOR for father's education and household income was 0.553 and 0.529 , respectively. This indicated that children who had one standard deviation higher in father's education or log-household income is 0.553 or 0.529 times less likely to be LBC than those in lower values when other independent variables in Model 4 were held constant. Since these values are similar and close to the threshold 0.50 , we could say that both father's education and household income are strong protective factors with approximately equal effects. At the community level, the mean father's education was statistically significant predictor and its SOR would be 0.373 , which indicated that children living in community with one SD unit 
higher in mean father's education is 0.373 times less likely to be LBC compared to those living in community with lower mean father's education value, holding other variables in the model constant.

Using the OR and SOR, we can compare the relative importance of independent variables within and across communities. Among six variables (Minority, $F_{-} E d$, and Log_Income at child/family level and Mean_Minority, Mean_F_Ed, and Mean_Income at community level), the most substantively important variable was Minority $(\mathrm{OR}=4.655)$, followed by Mean_F_Ed $(\mathrm{SOR}=0.373)$, and then Log_Income $(\mathrm{SOR}=0.529)$ and $F_{-} E d(\mathrm{SOR}=$ 0.553 ) with approximately equal importance. All of these values exceeded or were close to the threshold values of large ES (2.0 or 0.5), they can be considered to have substantively important large effects on LBC occurrence. The remaining Mean_Minority and Mean_Income were not statistically nor substantively significant when other variables in the model were held constant.

\section{Discussion}

The current study used the multilevel logistic regression model to explore what factors could predict the status of left behind children (LBC) in China using the 2011 CHNS data. In order to study the LBC occurrence, families with children under age 18 were selected from the original data set. The major findings are summarized in the following.

1. At the household/family level, father's educational attainment was the relevant parental education variable to the LBC occurrence, but mother's and parents' educational attainment were not.

2. At the household/family level, higher father's educational attainment and higher household income worked as the strong protective factors for the LBC occurrence with approximately equal effect size (ES).

3. At the community level, higher mean father's education was a strong protective factor, but the mean income was irrelevant. Mean minority was also not the significant predictor holding other variables constant.

4. Among the four variables of our research interest in the present study, i.e., father's educational attainment, household income at family level, and mean father's education and mean household income at community level, the strongest factor was the mean father's education, followed by household income and father's education with similar degree of effect size. All of them were strong protective factors for LBC. On the other hand, community mean household income was not relevant factor once other factors were held constant.

5. Though it was not statistically significant, the minority status of children had the largest ES among all the predictors considered, implying that it could be the strongest risk factor that leads to LBC.

6. There was large difference of proportion of LBC among five municipal/geographical regions. Among them, southwest region had significantly higher probability of LBC compared to other regions.

The first finding was indicated by the sensitivity analysis empirically, but it also fits to the rural family system in China conceptually. Even in modern times, the family culture inherited from a patriarchal society is still popular among rural areas in China, especially the villages with lower income and fewer resources. This family culture is symbolized as "man rules outside and woman rules inside", which expects the wife to be subservient to her husband under marital power. Thus, the patriarchal family culture that persists in rural China could have made the father's education a contributing factor that predicts the LBC occurrence in the present study.

In the second finding, that higher household income was a strong protective factor for LBC occurrence was consistent with the literature and our expectation. However, higher father's education as another strong protective factor was unexpected because many immigration studies in other countries indicated that the rural laborers with relatively high education were more likely to be employed in urban areas outside of their hometown (Van Ours and Veenman, 2003; Aydemir \& Sweetman, 2007; Aydemir, 2011).

Some scholars, however, indicated that although schooling is generally expected to promote job mobility and migration, it is not always reflected in Chinese data (Willmore, Cao, \& Xin, 2012). Zhu's (2002) study demonstrated that education level played a positive role in migration decision for men, but not for women. Other researchers even reported a weak effect of formal education on rural migration, but a strong effect for shifting from rural farm work to local non-farm work (Xiaoping, Heerink, \& Futian, 2007; Démurger, Fournier, \& Yang, 2010). The local off-farm work or employment such as rural village officers, educators, and doctors require employees to have higher educational attainment, and provide more stable and decent salaries than rural farm work. In such scenarios, higher educational attainment can indirectly play a protective role on alleviating the probability of LBC. 
Another possible explanation is that well educated fathers could better identify the detrimental effects this geographical separation may bring for the well-being of their children compared to fathers that are poorly educated. As suggested by Graham and Jordan (2011), a well-educated father may be more aware of the necessary role of parental companion on the childcare, having higher educational expectation from their children, and more concerned about children's spiritual or psychological needs during their growth. Therefore, even the labor migration could indeed improve the economic and nutritional circumstances for their children, the father with higher educational attainment may feel less willingly to migrate to urban areas for financial benefits at sacrifice of forming the stable attachment with their children and choose to stay with children by identifying off-farm work locally. It was highly likely that access to higher education may help them better understand the negative impacts that child separation from their parent(s) has on children's psychological well-being and their academic performance at schools (Wen \& Lin, 2012; Su et al., 2013; Hu, Lu, \& Huang, 2014).

For the third finding, we found that the mean father's educational attainment was a strong protective factor for the LBC, but the community average income was not. To understand why the average level of fathers' educational attainment within community could protect children from being left behind, we may turn our attention to the traditional rural culture in China. Traditionally, Chinese village fits Wolf's model of the closed corporate peasant community (Wolf, 1957). Within this enclosed community, people often perceived "guanxi", a typical Chinese word for the networks of personal connections, as a very foundation of the society in which they live. Through this "guanxi", people gradually build up intimate relationship and share common identity to guide each member's attitudes and behaviors. Therefore, with more well-educated fathers in the same community, the importance of parental care in children's wellbeing may be easily shared and supported through daily interaction among them. It then helped formulate a child-raising atmosphere as a community climate which emphasized the important role played by the parental companion at children' growth. This is a form of cultural capital or resources available in the community (Dubois, 2011).

Another portion of the third finding was unexpected. This result contradicted with the findings from other Chinese studies (Chan, 2013; Du, 2000; Yang, 2000), which concluded that the community capital income would significantly influence people's intention to migrate from low-income community to high-income community. Right now, it is unclear about why this happened to the current study. One reason for this discrepancy could be that most of these studies used a broader migration sample that did not distinguish individual and family migration. This could have masked the potential effect of having children at home on the decision to migrate to the urban areas for the employment. The result from the current study may imply that family life stage and characteristics could make the same factor play a different role in immigration decision, and family immigration decision may be made independently of local economy. However, the further study is warranted to see if the results of the present study can be replicated.

The fourth finding was about the relative strength of educational and economic factors that were working at different levels. The stark contrast between the mean father educational attainment being the strongest factor and no relevance of the mean income on the LBC occurrence could be one of the unique characteristics that Chinese rural villages have as a shared culture. At the child/family level, higher household income was a strong protective factor of the LBC occurrence, which was consistent with literature, and the present study revealed that the father's educational attainment was also a protective factor with approximately equal strength of impact.

The fifth finding was, though it was not statistically significant, being minority children were the largest risk factor of the LBC occurrence, but the proportion of minority was not related after other predictors such as mean father's education and geographical area were controlled for. The first part was consistent with literature except for the statistical significance (Zhao, 1999; Fan \& Zhao, 2010), but the second part was unexpected. These subtle findings require further examination in the future research.

The sixth finding about geographical regional difference on the probability of the LBC occurrence was consistent with literature. The Southwest area is the one that has highest proportion of left behind children, which is significantly higher than the Northeast and Costal region. The Southwest region in the current study included Guangxi and Guizhou, which is characterized as "mountains, steep slope, low water, valley deeps, thin soil, ground barren" with fragile ecological environment, harsh living space, undulating surface, rocky desertification and serious soil erosion. In addition to the harsh natural environment, these areas also have a large dense population with high proportion of weak education. These factors worked together to make the areas fall into the vicious circle of property. That is why these two provinces often have higher population of out-migrants than other provinces in China (Qin, 2010). 


\section{Implications of the Findings}

Based on the current findings, the lower household income is still a major determinant for the problem of LBC in China. This is consistant with other studies that emphasized the key role played by household income in their migration decision. In fact, increasing the household income in rural areas has been a long-term development goal for Chinese government, and the rural-urban income gap was remarkably narrowed from recent report (Mingmei, 2019). However, totally eliminating this income equality seems to be an impossible mission in the short term since a "dual system" that establishes the urban-rural divide is still existed in Chinese policy. The more practical solution in the short term is to increase the schooling opportunities for children with rural "hukou" so they can migrate with their parents to the destination cities. To accomplish this goal requires not only the deeper release of "hukou" registration system, but also more establishment of affordable public schools for the rural migrant children. This may be especially important for Preschoolers and adolescent children because they are particularly risky of being left behind (Tong, Yan., \& Kawachi, 2019).

The highlight of the fathers' education level in the current results also provided some insight into the intervention program targeted at the population of LBC in rural China. So far, many of them have been focusing on the facet of children's needs and using more passive strategies that only try to improve the well-beings of LBC (Guan \& Deng, 2019). Little attention has been given to establish more proactive or preventive programs targeted at the parents of LBC in rural China. Based on our findings about the important role played by father's education on the phenomenon of LBC in both micro and macro levels, it may be useful to organize intervention programs targeted to the parents, especially for the families where the fathers own lower education attainment. For example, one could develop community workshops or seminars that inform them of the trade-off between increased family income and disrupted parental care. This intervention may be especially important among the families where fathers did not have higher educational background in the communities where the mean father's education were relatively low. The goal of these programs is to raise their awareness of the detrimental effects of parental absence on children's emotional and psychological functioning.

\section{Limitations and Future Directions}

The targeted population in the current study was the families who already have their children in China. Therefore, it may be improper to generalize the current findings to broader migration population, especially for rural workers who do not have family. In fact, many empirical studies already found that higher education would increase the intention to migration among individual rural labors, and the more educated migrants tend to stay in urban area longer and have a higher chance of being employed in the manufacturing and service industries compared to the less educated migrates. This contradicting role played by male laborer's educational level on the intention to migration may imply that life or family stages may lead the same factor to function differently in the migration determination. In order to confirm this different role that the same variables could play requires further studies in the future.

The second limitation rests on the cross-sectional nature of the data. Since the current study used the cross-sectional data in 2011, which collected all the information in just one-time point. Therefore, the causal relationship cannot be inferred from our results. Further study should consider the longitudinal analysis to examine this phenomenon since there are higher chance to make causal inferences by having multiple waves of the same individuals in the same communities over time. Identifying causal factors would help come up an effective intervention program to alleviate this serious social problem.

\section{Acknowledgments}

We would like to acknowledge Dr. Michael Hughes, a professor in the Department of Sociology at Virginia Tech, for his hepful feedback to the earlier draft to improve the quality of the manuscript; Special thanks to $\mathrm{Ph}$. D candidate Kazuki Hori from Virgnia Tech for his contribution on merging different datasets.

\section{References}

Allison, P. D. (2009). Fixed effects regression models (Vol. 160). SAGE publications.

Aydemir, A. (2011). Immigrant selection and short-term labor market outcomes by visa category. Journal of Population Economics, 24(2), 451-475. https://doi.org/10.1007/s00148-009-0285-0

Aydemir, A., \& Sweetman, A. (2007). First-and second-generation immigrant educational attainment and labor market outcomes: A comparison of the United States and Canada. In Immigration (pp. 215-270). Emerald Group Publishing Limited. https://doi.org/10.1016/S0147-9121(07)00006-4

Borjas, G. J. (1993). The intergenerational mobility of immigrants. Journal of Labor Economics, 11(1, Part 1), 
113-135. https://doi.org/10.1086/298319

Cai, F., Du, Y., \& Wang, M. Y. (2009, April). Migration and labor mobility in China (Human development research paper). New York, NY: United Nations Development Programme.

Chan, J., \& Selden, M. (2014). China's rural migrant workers, the state, and labor politics. Critical Asian Studies, 46(4), 599-620._https://doi.org/10.1080/14672715.2014.960709

Chan, K. W. (2010). The global financial crisis and migrant workers in China: 'There is no future as a laborer; returning to the village has no meaning'. International Journal of Urban and Regional Research, 34(3), 659-677. https://doi.org/10.1111/j.1468-2427.2010.00987.x

Chan, K. W. (2013). China: internal migration. The encyclopedia of global human migration.

Chan, K. W., \& Buckingham, W. (2008). Is China abolishing the hukou system? The China Quarterly, 195, 582-606. https://doi.org/10.1017/S0305741008000787

Chen, A., \& Coulson, N. E. (2002). Determinants of urban migration: Evidence from Chinese cities. Urban Studies, 39(12), 2189-2197. https://doi.org/10.1080/0042098022000033818

Chinese Center of Disease Control and Prevention. (2019). China health and nutrition survey. Retrieved from https://www.cpc.unc.edu/projects/china

Dai, Q., \& Chu, R. X. (2018). Anxiety, happiness and self-esteem of western Chinese left-behind children. Child abuse \& neglect, 86, 403-413. https://doi.org/10.1016/j.chiabu.2016.08.002

De Haas, H. (2010). Migration and development: A theoretical perspective. International migration review, 44(1), 227-264. https://doi.org/10.1111/j.1747-7379.2009.00804.x

Démurger, S., Fournier, M., \& Yang, W. (2010). Rural households' decisions towards income diversification: Evidence from a township in northern China. China Economic Review, 21, S32-S44. https://doi.org/10.1016/j.chieco.2010.05.007

$\mathrm{Du}, \mathrm{Y}$. (2000). Rural labor migration in contemporary China: an analysis of its features and the macro context. Rural Labor Flows in China, Institute of East Asian Studies, University of California, Berkeley.

Dubois, V. (2011). Cultural capital theory vs. cultural policy beliefs: How Pierre Bourdieu could have become a cultural policy advisor and why he did not. Poetics, 39(6), 491-506. https://doi.org/10.1016/j.poetic.2011.09.003

Ebenstein, A., \& Zhao, Y. (2015). Tracking rural-to-urban migration in China: Lessons from the 2005

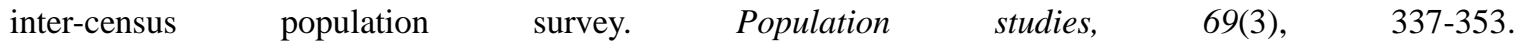
https://doi.org/10.1080/00324728.2015.1065342

Fan, C. C. (2002). The elite, the natives, and the outsiders: Migration and labor market segmentation in urban China. Annals of the Association of American Geographers, 92(1), 103-124. https://doi.org/10.1111/1467-8306.00282

Fan, L., \& Zhao, W. (2010). The survey for left-behind children's life satisfaction. Journal of Educational Development, 2, 42-44.

Fan, F., Su, L., Gill, M. K., \& Birmaher, B. (2010). Emotional and behavioral problems of Chinese left-behind children: A preliminary study. Social psychiatry and psychiatric epidemiology, 45(6), 655-664. https://doi.org/10.1007/s00127-009-0107-4

Gao, W., \& Smyth, R. (2011). What keeps China's migrant workers going? Expectations and happiness among China's floating population. Journal of the Asia Pacific Economy, 16(2), 163-182. https://doi.org/10.1080/13547860.2011.564749

Ge, L. (2019). A Review of the Phenomenon of Left-behind Children in China from the Perspective of Historicism with Discussion and Analysis of the Relevant Research Literature. Journal of Studies in Social Sciences, 18(2). https://doi.org/10.28924/ip/jsss.1917

Graham, E., \& Jordan, L. P. (2011). Migrant parents and the psychological well-being left-behind children in $\begin{array}{lllll}\text { Southeast Asia. Journal of Marriage and Family, 73(4), } & \text { 763-787. }\end{array}$ https://doi.org/10.1111/j.1741-3737.2011.00844.x

Guan, S., \& Deng, G. (2019). Whole-community intervention for left-behind children in rural China. Children and Youth Services Review, 101, 1-11. https://doi.org/10.1016/j.childyouth.2019.03.033 
Hare, D. (1999). 'Push' versus 'pull' factors in migration outflows and returns: Determinants of migration status and spell duration among China's rural population. The Journal of Development Studies, 35(3), 45-72. https://doi.org/10.1080/00220389908422573

Hu, H., Lu, S., \& Huang, C. C. (2014). The psychological and behavioral outcomes of migrant and left-behind children in China. Children and Youth Services Review, 46, 1-10. https://doi.org/10.1016/j.childyouth.2014.07.021

Huang, Y. (2014). The continuity and changes of the hukou system since the 1990s: A critical review. In Urban China in the New Era (pp. 25-43). Springer Berlin Heidelberg. https://doi.org/10.1007/978-3-642-54227-5_2

Jia, Z., \& Tian, W. (2010). Loneliness of left-behind children: a cross-sectional survey in a sample of rural China. Child: care, health and development, 36(6), 812-817. https://doi.org/10.1111/j.1365-2214.2010.01110.x

Kaufman, R. (1996). Comparing Effects in Dichotomous Logistic Regression: A Variety of Standardized Coefficients. Social Science Quarterly, 77(1), 90-109. Retrieved August 26, 2020, from http://www.jstor.org/stable/42863433

Kuang, L., \& Liu, L. (2012). Discrimination against rural-to-urban migrants: the role of the hukou system in China. PloS one, 7(11), 46932. https://dx.doi.org/10.1371\%2Fjournal.pone.0046932

Lee, M. H. (2011). Migration and children's welfare in China: The schooling and health of children left behind. The Journal of Developing Areas, 44(2), 165-182. Retrieved from http:/www.jstor.org/stable/23215246

Li, H., \& Zahniser, S. (2002). The determinants of temporary rural-to-urban migration in China. Urban Studies, 39(12), 2219-2235. https://doi.org/10.1080\%2F0042098022000033836

Liang, Z., \& Chen, Y. P. (2004). Migration and gender in China: An origin-destination linked approach. Economic Development and Cultural Change, 52(2), 423-443. https://doi.org/10.1086/380594

Liang, Z., \& Ma, Z. (2004). China's floating population: new evidence from the 2000 census. Population and development review, 30(3), 467-488. https://doi.org/10.1111/j.1728-4457.2004.00024.x

Liu, S., Xie, F., Zhang, H., \& Guo, S. (2014). Influences on rural migrant workers' selection of employment location in the mountainous and upland areas of Sichuan, China. Journal of Rural Studies, 33, 71-81. https://doi.org/10.1016/j.jrurstud.2013.11.001

Mallee, H., \& Pieke, F. N. (2014). Internal and international migration: Chinese perspectives. Routledge.

Massey, D. S. (1990). 1990: Social structure, household strategies, and the cumulative causation of migration. Population Index, 56, 3-26. https://doi.org/10.2307/3644186

Mejı, A., Pizurki, H., Royston, E., \& World Health Organization. (1979). Physician and Nurse Migration: Analysis and Policy Implications: Report of a WHO Study. Geneva: World Health Organization.

Mimi L. (2016, October 21). China has 61 million left-behind children ... that's almost Britain's total population. South China Morning Post. Retrieved from https://www.scmp.com/news/china/society/article/2038731/ china-has-61-million-left-behind-children-thats-almost-britains

Mingmei. (2019, August 8). China sees rapid income, consumption growth in rural areas over past 70 years. Xinhuanet. Retrieved from http://www.xinhuanet.com/english/2019-08/08/c_138293816_2.htm

Monahan, P. O., McHorney, C. A., Stump, T. E., \& Perkins, A. J. (2007). Odds ratio, delta, ETS classification, and standardization measures of DIF magnitude for binary logistic regression. Journal of Educational and Behavioral Statistics, 32(1), 92-109. https://doi.org/10.3102\%2F1076998606298035

Morawska, E. (2007). International migration: Its various mechanisms and different theories that try to explain it. Willy Brandt Series of Working Papers in International Migration and Ethnic Relations. Retrieved from http://urn.kb.se/resolve?urn=urn:nbn:se:mau:diva-12919

Ours, J. C., \& Veenman, J. (2003). The educational attainment of second-generation immigrants in The Netherland. Journal of Population Economics, 16(4), 739-753. https://doi.org/10.1007/s00148-005-0055-6

Qin, H. (2010). Rural-to-urban labor migration, household livelihoods, and the rural environment in Chongqing Municipality, Southwest China. Human Ecology, 38(5), 675-690. https://doi.org/10.1007/s10745-010-9353-z

Qu, G. B., Wu, W., Wang, L. L., Tang, X., Sun, Y. H., Li, J., \& Wang, J. (2018). Systematic review and metaanalysis found higher levels of behavioural problems in male left-behind children aged 6-11 years. Acta 
Paediatrica, 107(8), 1327-1334. https://doi.org/10.1111/apa.14199

Raudenbush, S. W., \& Bryk, A. S. (2002). Hierarchical linear models: Application and data analysis methods. Thousand Oaks, CA: Sage.

Raudenbush, S. W., Bryk, A. S, Cheong, Y. F., Congdon, R., \& du Toit, M. (2011). HLM 7 for Windows [Computer software]. Skokie, IL: Scientific Software International, Inc.

Roberts, K. D. (2001). The determinants of job choice by rural labor migrants in Shanghai. China Economic Review, 12(1), 15-39. https://doi.org/10.1016/S1043-951X(01)00041-4

Shao, C., Meng, X., Cui, S., Wang, J., \& Li, C. (2016). Income-related health inequality of migrant workers in China and its decomposition: an analysis based on the 2012 China labor-force dynamics survey data. Journal of the Chinese Medical Association, 79(10), 531-537. https://doi.org/10.1016/j.jcma.2016.02.009

Shi-tan, D. O. N. G. (2009). An Analysis of Crimes Committed by Left-behind Children in the Countryside of Shandong Province. Journal of Shandong Police College, 4.

Singer, J. D., \& Willett, J. B. (2003). Applied longitudinal data analysis: Modeling change and event occurrence. Oxford University Press. https://doi.org/10.1093/acprof:oso/9780195152968.001.0001

Su, S., Li, X., Lin, D., Xu, X., \& Zhu, M. (2013). Psychological adjustment among left-behind children in rural China: The role of parental migration and parent-child communication. Child: Care, health and development, 39(2), 162-170. https://doi.org/10.1111/j.1365-2214.2012.01400.x

Swain, L. L., \& Garasky, S. (2007). Migration decisions of dual-earner families: An application of multilevel modeling. Journal of Family and Economic Issues, 28(1), 151-170. https://doi.org/10.1007/s10834-006-9046-3

Tong, Y., \& Piotrowski, M. (2012). Migration and health selectivity in the context of internal migration in China, 1997-2009. Population Research and Policy Review, 31(4), 497-543. https://doi.org/10.1007/s11113-012-9240-y

Tong, L., Yan, Q., \& Kawachi, I. (2019). The factors associated with being left-behind children in China: Multilevel analysis with nationally representative data. PloS one, 14(11). https://doi.org/10.1371/journal.pone.0224205

Wen, M., \& Lin, D. (2012). Child development in rural China: Children left behind by their migrant parents and children of nonmigrant families. Child development, 83(1), 120-136. https://doi.org/10.1111/j.1467-8624.2011.01698.x

Willmore, L., Cao, G. Y., \& Xin, L. J. (2012). Determinants of off-farm work and temporary migration in China. Population and Environment, 33(2-3), 161-185. https://doi.org/10.1007/s11111-011-0135-3

Wolf, E. R. (1957). Closed corporate peasant communities in Mesoamerica and Central Java. Southwestern Journal of Anthropology, 13(1), 1-18. Retrieved August 26, 2020, from http://www.jstor.org/stable/3629154

Xiaoping, S. H. I., Heerink, N., \& Futian, Q. U. (2007). Choices between different off-farm employment sub-categories: An empirical analysis for Jiangxi Province, China. China Economic Review, 18(4), 438-455. https://doi.org/10.1016/j.chieco.2006.08.001

Yang, X. (2000). Determinants of migration intentions in Hubei province, China: individual versus family migration. Environment and Planning A, 32(5), 769-787. https://doi.org/10.1068\%2Fa32114

Yang, X., \& Guo, F. (1999). Gender differences in determinants of temporary labor migration in China: A multilevel analysis. International Migration Review, 33(4), 929-953. https://doi.org/10.1177\%2F019791839903300405

Ye, J., \& Lu, P. (2011). Differentiated childhoods: Impacts of rural migration on left-behind children in China. The Journal of peasant studies, 38(2), 355-377. https://doi.org/10.1080/03066150.2011.559012

Ye, J., Wang, C., Wu, H., He, C., \& Liu, J. (2013). Internal migration and left-behind populations in China. Journal of Peasant Studies, 40(6), 1119-1146. https://doi.org/10.1080/03066150.2013.861421

Zhang, J. F., Xie, F. T., Liu, S. Q., Cao, M. T., \& Liu, E. L. (2015). Influential factors in employment location selection based on "push-pull" migration theory: A case study in Three Gorges Reservoir area in China. Journal of Mountain Science, 12(6), 1562-1581. https://doi.org/10.1080/03066150.2013.861421

Zhao, Q., Yu, X., Wang, X., \& Glauben, T. (2014). The impact of parental migration on children's school performance in rural China. China Economic Review, 31, 43-54. 
https://doi.org/10.1016/j.chieco.2014.07.013

Zhao, Y. (1999). Leaving the countryside: Rural-to-urban migration decisions in China. American Economic Review, 89(2), 281-286. https://doi.org/10.1257/aer.89.2.281

Zhou, H., \& Zhu L. (2019). "Two Chinas": Social Equity, Social Policies, and the Urban-Rural Divide in China. In M. Johansen (Ed.), Social Equity in the Asia-Pacific Region. Palgrave Macmillan, Cham. https://doi.org/10.1007/978-3-030-15919-1_7

Zhu, N. (2002). The impacts of income gaps on migration decisions in China. China Economic Review, 13(2), 213-230. https://doi.org/10.1016/S1043-951X(02)00074-3

\section{Notes}

Note 1. This value 0.298 from the HGLM is slightly lower than 0.356 , the simple observed proportion. This is because the HGLM estimates the unit specific fixed effects as opposed to the population average estimate which can be estimated by generalized estimating equation (GEE) methodology. It is common to observe the slightly lower estimate for the HGLM unit specific model than the population average estimate (Chapter 10, Raudenbush \& Bryk, 2002).

Note 2. It may be more appropriate to refer to semi-standardized or $\mathrm{x}$-standardized odds-ratio since only the independent variable is standardized. However, for simplicity, we refer it to the standardized odds-ratio (SRO) in this article.

\section{Copyrights}

Copyright for this article is retained by the author(s), with first publication rights granted to the journal.

This is an open-access article distributed under the terms and conditions of the Creative Commons Attribution license (http://creativecommons.org/licenses/by/4.0/). 